\title{
ANÁLISIS TEÓRICO DE LAS ECUACIONES FUNDAMENTALES DEL PROCESO DE LA DIFRACCIÓN DE ELECTRONES RETRODISPERSADOS Y SU APLICACIÓN EN CIENCIA DE LOS MATERIALES \\ THEORETICAL ANALYSIS OF FUNDAMENTAL EQUATIONS OF ELECTRONS BACKSCATTER DIFFRACTION AND ITS APPLICATION IN THE SCIENCE OF MATERIALS
}

\author{
José Antonio Tiburcio Moreno', Pelayo Delgado Tello²
}

\section{RESUMEN}

El proceso de difracción de electrones retrodispersados es una técnica basada en el análisis de las bandas de Kikuchi, determinadas por la excitación de un haz de electrones sobre la superficie de una muestra en estado sólido, mediante esta interacción es posible determinar la estructura cristalina, orientación de los planos del cristal y la microestructura de forma más detallada respecto a otras técnicas. También, es posible observar en tiempo real los tipos de frontera de granos y las fases de la superficie de los materiales, siendo estos parámetros de vital importancia en el diseño y fabricación de materiales para aplicaciones industriales.

Palabras clave: Electrones retrodispersados, bandas de Kikuchi, estructura cristalina, microestructura, frontera de granos.

\section{ABSTRACT}

The backscattered electrons diffraction process is a technique based on the Kikuchi bands analysis, determined by the excitation of an electron beam on the surface of a solid state sample, through this interaction it is possible to determine the structure crystalline orientation of the glass planes and microstructure in more detail with respect to other techniques. It is also possible to observe in real time the types of grain boundaries and the surface phases of the materials, these parameters being of vital importance in the design and manufacture of materials for industrial applications.

Key words: Backscattered electrons, Kikuchi bands, crystal structure, microstructure, grain boundaries.

\section{INTRODUCCIÓN}

La difracción de electrones retrodispersados (siglas en ingles EBSD) es una técnica que permite obtener información cristalográfica de muestras a través de un microscopio electrónico de barrido (siglas en ingles SEM). En la técnica EBSD un haz de electrones estacionario golpea una muestra cristalina y los electrones difractados forman un patrón en una pantalla fluorescente. Este patrón de difracción es característico de la estructura cristalina y de la orientación de la región de muestra desde donde se generó. El patrón de difracción puede ser usado para medir la orientación del cristal, las orientaciones de los límites de grano de la muestra, discriminar la estructura entre diferentes materiales y proporcionar información sobre la perfección del material. Cuando el haz es escaneado en una cuadrícula a través de una

\footnotetext{
1 Doctor en Ciencias en la especialidad de Física Aplicada

Docente de la Facultad de Ciencias - Universidad Nacional Jorge Basadre Grohmann - Tacna Perú

2 Doctor en Economía

Docente de la Facultad de Ciencias - Universidad Nacional Jorge Basadre Grohmann - Tacna Perú
} 
muestra policristalina y la orientación del cristal es medida en cada punto, el mapa resultante revelará los constituyentes de la morfología del grano, las orientaciones y límites de los constituyentes de la muestra. Esos datos también se pueden utilizar para mostrar las orientaciones cristalinas la cual está ligada a la textura del material. En este sentido una completa y cuantitativa representación de la muestra cristalina se puede obtener mediante esta técnica.

\section{Componentes Principales del Sistema EBSD}

Los principales componentes del sistema EBSD se muestran en la Figura 1:

$>$ La muestra está inclinada a $70^{\circ}$ de la horizontal.

> Una pantalla fosforescente es impactada por los electrones que se desvían al impactar sobre la muestra para formar un patrón de difracción.

$>$ Un detector CCD sirve para visualizar el patrón de difracción en la pantalla.

$>$

> Sistema electrónico para controlar el SEM, incluido la posición del haz de electrones, posición de las lentes y la magnificación.

$>$ Un control computarizado analiza los experimentos de EBSD a través de los patrones de difracción y muestra los resultados.

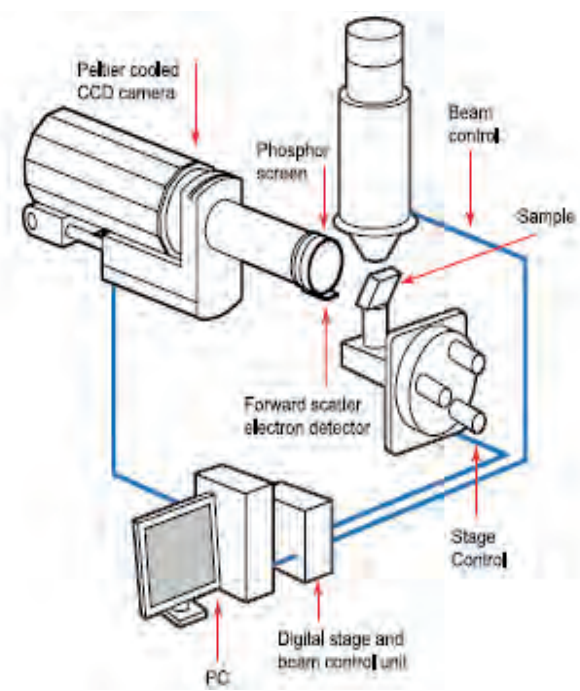

Figura 1: Esquema de un Microscopio Electrónico de Barrido (SEM) y el sensor EBSD acoplado en su cámara de detección.

\section{Formación y del patrón de difracción}

El haz de electrones es dirigido a un punto de interés sobre una muestra inclinada situada en un Microscopio Electrónico de Barrido (SEM, ver Figura 1). El mecanismo por el cual el patrón de difracción se forma es complejo, pero el siguiente modelo describe las principales características. Los átomos en el material dispersan inelásticamente una fracción de los electrones incidentes, los cuales pierden una pequeña cantidad de energía para formar una fuente divergente de electrones cerca de la superficie de la muestra. Algunos de estos electrones inciden sobre planos atómicos en ángulos que satisfacen la bien conocida ecuación de Bragg (ecuación 1).

$$
2 d \operatorname{sen} \theta=n \lambda
$$

Donde $d$ es la distancia interplanar de la estructura cristalina, $\theta$ es el ángulo de incidencia de la radiación sobre la muestra, $\lambda$ es la longitud de onda de la radiación incidente y $n$ es el orden de difracción.

Estos electrones son difractados para formar un conjunto de ángulos cónicos que corresponden a cada plano de difracción, los cuales determinan una imagen en la pantalla fluorescente, las regiones de mayor intensidad entre los conos producen las características bandas de Kikuchi de los electrones retrodispersados (ver figuras 2 , 3 y 4 )

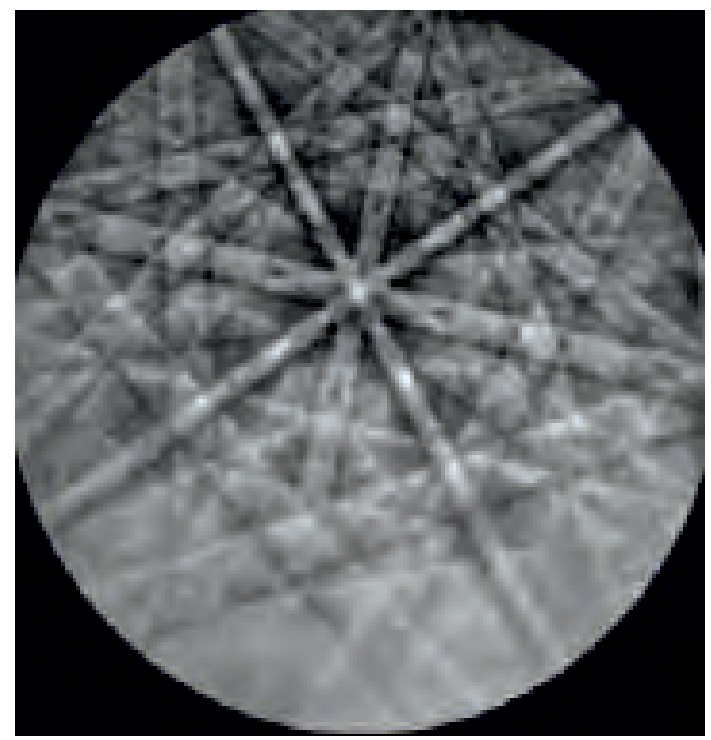

Figura 2: Patrón de difracción típico de un metal 

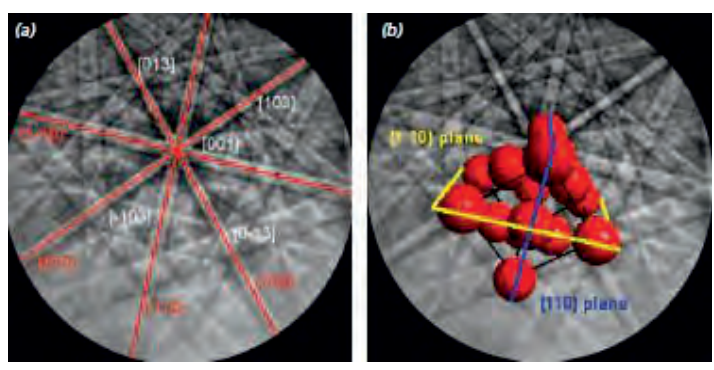

Figura 3: (a) Patrón de difracción indexado (figura 2 ), las bandas de Kikuchi están nominadas con los índices de Miller de los planos cristalinos que las forman (rojas) y los puntos de cruce de las bandas son denominados como ejes (blanco), (b) orientación de la celda unitaria la cual genera el patrón de difracción con sus correspondientes planos mostrados.

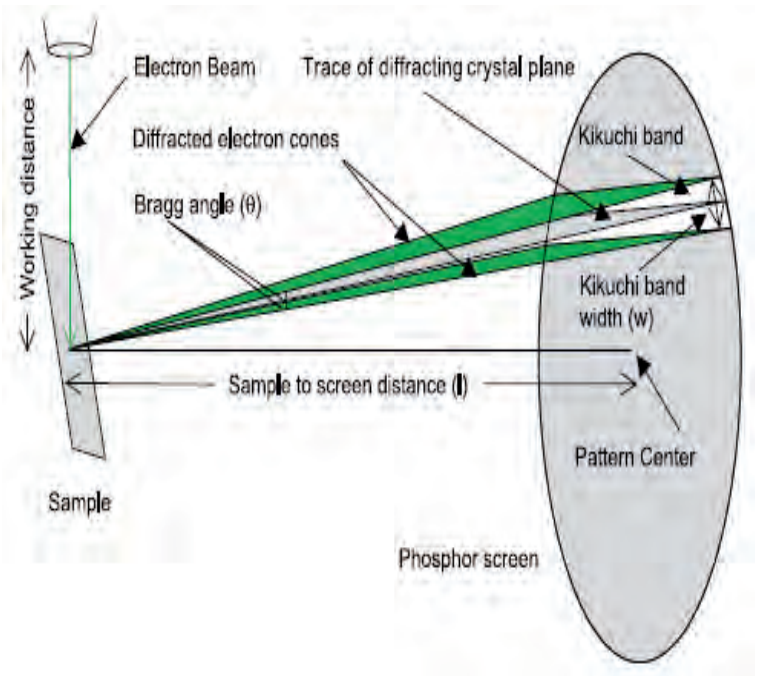

Figura 4: Geometría de la técnica EBSD

\section{MATERIALES Y MÉTODO}

- Sensor fotosensible acoplado a un Microscopio Electrónico de Barrido

- Tarjeta de adquisición de datos acoplada desde una computadora al SEM

- Software Aztec para analizar los patrones de difracción

- Muestras metálicas

\section{RESULTADOS}

Resultados experimentales para una muestra cerámica, el software asociado al sensor EBSD del microscopio electrónico genera las indexaciones basadas en la ley de Bragg y es posible obtener la estructura cristalográfica realizando la correspondiente comparando con bases de datos estándar.
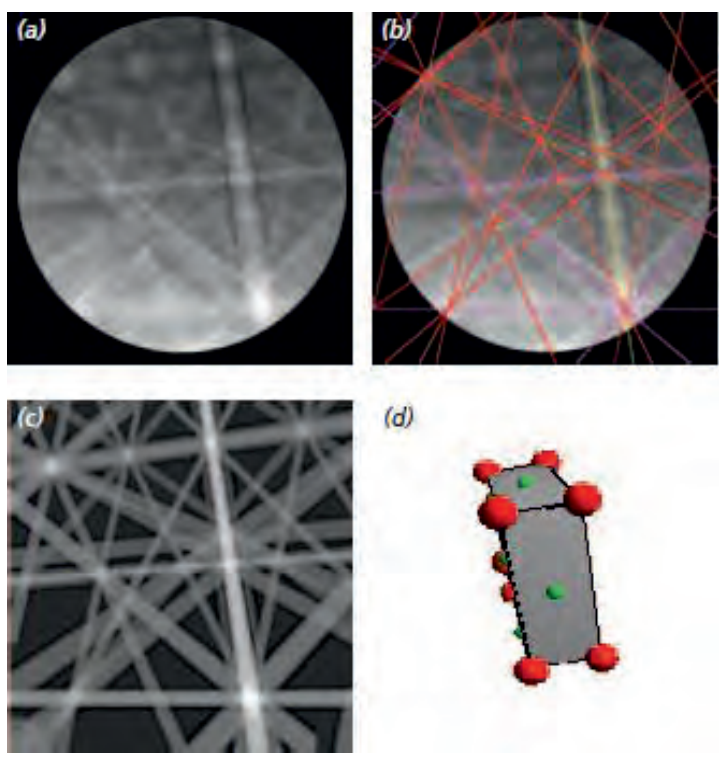

(d)

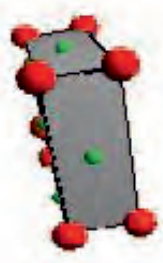

Figura 5: Patrón de difracción de intensidades de bandas, (a) patrón de difracción de una cerámica ortorrómbica, (b) soluciones superpuestas de patrón de difracción dado por la orientación del cristal, (c) patrón de difracción simulado mostrando las bandas de Kikuchi, (d) simulación de la orientación cristalina.

\section{DISCUSIÓN}

\section{Interpretación de los Patrones de Difracción}

Las líneas centrales de las bandas de Kikuchi corresponden a las intersecciones de los planos de difracción con la pantalla fosforescente. Entonces, cada banda de Kikuchi puede ser indexada por los índices de Miller de los planos cristalinos de difracción que las forman.

Las intersecciones de las bandas de Kikuchi corresponden a los ejes en el cristal. El semi ángulo de los conos de difracción de electrones es $(90-\theta)$ grados. Pero en EBSD este es un ángulo muy grande de modo que, las bandas de Kikuchi se aproximan a líneas rectas. Por ejemplo, la longitud de onda de electrones de 20 $\mathrm{keV}$ es $0,00859 \mathrm{~nm}$ y el espaciamiento del plano (111) en el Aluminio es $0,233 \mathrm{~nm}$ haciendo un semi ángulo de $88,9^{\circ}$. El ancho $w$ de las bandas de Kikuchi cerca al centro del patrón de difracción es dado por:

$$
\omega=2 l \theta=\frac{n l \lambda}{d}
$$

Donde I es la distancia desde la muestra a la pantalla (figura 4). Asi los planos con amplio espaciamiento $d$ dan bandas de Kikuchi más 
delgadas que los planos con menos espaciamiento $d$. debido a que el patrón de difracción está enlazado a la estructura cristalina de la muestra, cuando la orientación del cristal cambia el patrón de difracción resultante también debe cambiar. Las posiciones de las bandas de Kikuchi pueden ser usadas para calcular las orientaciones de la difracción cristalina.

\section{Mediciones de la orientación e indexación}

La orientación del cristal es calculada por las posiciones de las bandas de Kikuchi mediante un procesamiento computarizado, el patrón de difracción colectado por la cámara CCD es digitalizado, luego las posiciones de las bandas de Kikuchi se encuentran usando la Transformada de Hough, que consiste en la transformación entre las coordenadas $(x, y)$ del patrón de difracción y las coordenadas $(\rho, \theta)$ en el espacio de Hough la cual es realizada mediante la siguiente ecuación:

$$
\rho=x \cos \theta+y \operatorname{sen} \theta
$$

Una línea recta es caracterizada por $\rho$, la distancia perpendicular desde el origen a un ángulo $\theta$ es representado por un simple punto $(\rho, \theta)$ en el espacio de Hough (Figura 6).
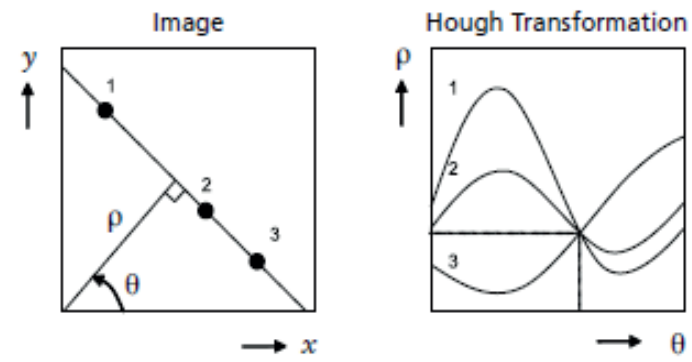

Figura 6: La Transformada de Hough convierte líneas en puntos en el espacio de Hough.

Las bandas de Kikuchi se transforman regiones de brillo intenso en el espacio de Hough, las cuales pueden ser detectadas y usadas para calcular las posiciones originales de las bandas.

\section{Intensidad de las Bandas}

Los mecanismos que dan lugar a las intensidades de las bandas de Kikuchi y las formas de sus perfiles son complejas, sin embargo, se puede aproximar la intensidad de una banda de intensidad $I_{h k l}$ para el plano (hkl) es dada por:

$$
\begin{gathered}
I_{h k l}=\left[\sum_{i} f_{i}(\theta) \cos 2 \pi\left(h x_{i}+k y_{i}+l z_{i}\right)\right]^{2}+ \\
{\left[\sum_{i} f_{i}(\theta) \operatorname{sen} 2 \pi\left(h x_{i}+k y_{i}+l z_{i}\right)\right]^{2}}
\end{gathered}
$$

Donde $f_{i}(\theta)$ es el factor atómico de difracción para electrones y $\left(x_{i}, y_{i}, z_{i}\right)$, son las coordenadas fraccionales en la celda unidad para el átomo $i$. Un patrón de difracción observado puede ser comparado con una simulación usando la ecuación anterior, para asegurar que solamente planos que producen bandas de Kikuchi visibles son usadas cuando se resuelve el patrón de difracción, esto es especialmente importante cuando se trabaja con materiales de más de un tipo de átomos.

\section{CONCLUSIONES}

1. Cuando un haz de electrones incide sobre una muestra inclinada montada en un SEM, se pueden generar electrones retrodispersados los cuales son detectados a través de un patrón de difracción sobre una pantalla fluorescente, a partir del cual se pueden determinar diferentes propiedades de una estructura cristalina.

2. La línea central de cada banda de Kikuchi corresponde a la intersección con la pantalla fluorescente del plano de difracción que determina la banda.

3. La posición de las bandas de Kikuchi pueden ser encontradas mediante la Transformada de Hough y usada para calcular la orientación cristalina de la región de la muestra que originó el patrón de difracción.

4. Mediante la ecuación (4) es posible realizar un proceso de simulación numérico para determinar la posición de las bandas de Kikuchi y poder estimar los parámetros asociados a ellas.

\section{REFERENCIAS BIBLIOGRÁFICAS}

HEIDENREICH, ROBERT D.A. KODAK., (2009), Fundamentals of transmission electron microscopy, Data Bok Inc., 179.

NIXON, W. C., (1969), Introduction to Scanning Electron Microscopy, NT Research Institute, Vol. 10, n. 2. 
DYKSTRA,M.,(1992), Biological electron microscopy: theory, techniques and troubleshooting, plenum Press.

HAYAT, M., (1981), Fixation for electron microscopy, Academic Press

M. L. LÓPEZ., (2003), Serie Científica Avanzada: El microscopio Electrónico, Centro de Extensión Biomédica Facultad de Medicina Universidad de Chile.

Oxford Instrument microanalysis group (1997), Link ISIS operator's guide, Oxford instruments (UK).

POBLETE, VICTOR, P.M. PILLEUX C., (2001),
Ciencia De Los Materiales "Microscopía Electrónica De Barrido", Universidad de Chile. Facultad de Ciencias Físicas y Matemáticas.

KLUG, H. P., (1954), X-Ray Diffraction Procedures for polycrystalline and amorphous materials: New York, John Wiley and Sons, 716 p.

WAGNER, T., WENZEL, T., (2007), Gold upgrading in metamorphosed massive sulfide ore deposits: Direct evidence from laserablation-inductive coupled plasma-mass spectrometry analysis of invisible gold: Geology, $35,775-778$. 